Revue d'histoire de l'Amérique française

FQ REVUE D.HISTOIRE DE L'AMÉRIQUE FRANÇAISE

\title{
Les questions internationales dans les premiers inédits de Lionel Groulx (1895-1909)
}

\section{Réjean Bergeron et Yves Drolet}

Volume 34, numéro 2, septembre 1980

URI : https://id.erudit.org/iderudit/303858ar

DOI : https://doi.org/10.7202/303858ar

Aller au sommaire du numéro

Éditeur(s)

Institut d'histoire de l'Amérique française

ISSN

0035-2357 (imprimé)

1492-1383 (numérique)

Découvrir la revue

Citer cet article

Bergeron, R. \& Drolet, Y. (1980). Les questions internationales dans les premiers inédits de Lionel Groulx (1895-1909). Revue d'histoire de l'Amérique française, 34(2), 245-255. https://doi.org/10.7202/303858ar d'utilisation que vous pouvez consulter en ligne. 


\title{
LES QUESTIONS INTERNATIONALES DANS LES PREMIERS INÉDITS DE LIONEL GROULX (1895-1909)
}

\author{
RÉJEAN BERGERON \\ YVES DROLET \\ Édition critique de Groulx \\ Université de Montréal
}

\section{Introduction}

Il peut sembler paradoxal de traiter des questions internationales dans l'oeuvre d'un penseur aussi intensément nationaliste que Lionel Groulx (1878-1967). Pourtant, dès la fin du XIXe siècle, Groulx manifeste une vive curiosité à l'égard des problèmes internationaux et situe la marche historique du Canada français dans un contexte mondial ${ }^{1}$. On peut donc se demander dans quelle mesure l'idée qu'il se faisait de son peuple et l'image qu'il avait du monde extérieur s'influencèrent l'une l'autre et jusqu'à quel point les deux reflétaient sa vision du monde.

La lecture des inédits de jeunesse de Groulx apporte des éléments de réponse à ces deux questions. En effet, le Journal intime, rédigé de 1895 à 1904, est parsemé de commentaires et d'annotations qui permettent de reconstruire l'image que Groulx s'était faite d'un monde qu'il ne connaissait que par les livres. D'autre part, les Notes et souvenirs de mon voyage en Europe (1906-1909) et la correspondance de l'époque permettent de mesurer l'étendue et les limites de l'impact qu'a pu avoir sur cette image un contact avec les

1 En 1900, il publie trois articles dans Le Salaberry de Valleyfield sur des questions d'actualité internationale: «Angleterre ou Russie?», 17 juillet; «En Chine, les causes de la crise», 31 juillet; «Le Vatican et l'assassinat d'Humbert», 14 août. Dans les trois cas, il analyse les événements en fonction du catholicisme et de l'intervention des forces surnaturelles dans l'histoire humaine.

RHAF, vol. 34, no 2, septembre 1980 
réalités européennes ${ }^{2}$. C'est à partir de ces textes que nous allons tenter de décrire les grands traits d'une vision du monde que le grand historien de l'Amérique française pourra certes réajuster, sans jamais toutefois la modifier en profondeur.

\section{1- Lionel Groulx et les questions internationales (1895-1904)}

C'est en 1895 que s'anime pour la première fois la plume qui, pendant trois quarts de siècle, allait tracer et retracer l'histoire du Canada français. Élève de belles-lettres au Séminaire de SainteThérèse, Lionel-Adolphe Groulx a dix-sept ans et commence à rédiger son Journal intime en s'inspirant du journal romantique d'Eugénie de Guérin, femme de lettres française; son premier acte d'écriture est déjà placé sous le signe de l'Europe.

L'Europe est alors au faîte de sa puissance et il ne faut pas s'étonner que la vision du monde de Groulx gravite autour du Vieux Continent. Les auteurs qui lui inspirent cette vision sont d'ailleurs presque tous européens: en 1895, il écrit: «Lamennais, Veuillot, les Guérin, O’Connell, tels sont mes héros! $»^{3}$ En 1896, il découvre: «...un bien grand homme, un prince des orateurs, un jurisconsulte célèbre, un grand Français: Berryer. ${ }^{4}$ À la même époque, il se passionne pour Lacordaire et Montalembert dont il fera les modèles à suivre pour les jeunes de l'Action catholique. En 1899, il note: «Je suis à lire le «Génie du Christianisme» de Chateaubriand. Je n'avais encore rien lu de ce grand auteur. C'est une révélation pour moi.» ${ }^{5}$

Veuillot, Berryer, Lacordaire, auxquels nous pouvons ajouter Joseph de Maistre et Donoso Cortès, tels sont les auteurs catholiques traditionalistes du XIXe siècle auxquels Groulx emprunte l'essentiel de sa conception d'un monde, théâtre d'une lutte sans merci entre Dieu et Satan. Dans cette vision manichéenne, l'histoire de l'humanité se résume au combat du bien représenté par les nations catholiques contre le mal personnifié par leurs ennemis. Pour Groulx, il y a une Vérité, incarnée par l'Église catholique romaine dirigée par le pape, «figure dominante du monde» ${ }^{6}$. Cette Vérité est proposée par Dieu aux nations qu'il a créées: si elles l'acceptent,

2 L'édition de ces manuscrits a été confiée à une équipe de chercheurs de l'Université de Montréal. Le Journal intime et les Notes et souvenirs... doivent être publiés dans un avenir assez rapproché.

3 Lionel Groulx, Journal intime, cahier I, $10 \mathrm{~ms}$.

Ibid., cahier I, $19 \mathrm{~ms}$.

Ibid., cahier III, $132 \mathrm{~ms}$.

Ibid., cahier IV, $40 \mathrm{~ms}$. 
elles auront droit aux consolations divines au milieu des tribulations que ne manqueront pas de leur susciter les autres, celles qui pactisent avec le diable mais qui seront punies.

Ainsi, l'Église apprend aux Polonais catholiques opprimés par les Russes orthodoxes:

(...) que le triomphe du méchant se change bientôt en défaite et que l'opprimé devient le triomphateur. Oui la Pologne revivra. J'en ai l'espoir puisqu'un peuple qui garde sa foi ne saurait perır.'

Semblable à la Pologne, l'Irlande catholique résiste à l'Angleterre protestante à laquelle Groulx décoche une diatribe cinglante:

Je ne connais pas chez les barbares de l'Antiquité païenne de plus abominable, de plus satanique tyrannie que celle que l'Angleterre a fait peser sur l'Irlande, qui à l'heure même, est loin de jouir d'une liberté parfaite. Aussi j'ai fait des colères bleues contre Albion, et me voilà maintenant rempli de sympathie pour ces pauvres Irlandais. ${ }^{8}$

À la même époque, Groulx écrit pourtant au sujet d'une tragédie composée par un de ses professeurs mettant en scène le roi d'Angleterre Édouard le Confesseur ( + 1066):

Le drame faisait revivre la figure la plus sympathique, la plus chrétienne du trône d'Angleterre. Albion en était à son âge de lumiere et de foi, et Rome encore la couvrait de son égide immortelle. ${ }^{9}$

Il y a donc pour lui deux Angleterre: la bonne, celle du Moyen Age, catholique et juste, et l'autre, la protestante, qui a perdu la lumière et la foi et qui retombe par conséquent dans la barbarie paienne dont témoigne son traitement des Irlandais... et des Canadiens français.

De même, il y a deux France, la catholique, celle des croisés et des zouaves, et l'impie, celle des jacobins et des anti-cléricaux; à la différence de l'Angleterre où le mal a triomphé dès le XVIe siècle, la France connaît en ce tournant de siècle les affres du combat commencé en 1789. À la veille des élections de 1902 qui devaient porter Émile Combes au pouvoir, Groulx écrit:

J'ai aussi songé plusieurs fois aux élections de la France pour le succès desquelles nous prions tous ensemble. Pauvre France

Ibid., cahier I, $50 \mathrm{~ms}$.

8 Ibid., cahier I, 27-28 ms.

$9 \quad$ Ibid., cahier I, 74 ms. 
triomphera-t-elle de tous les sans-dieu et de tous les sans-patrie qui la gouvernent et la déchristianisent? (...) il faut bien le dire, la France par ses fautes a été l'Israël des temps modernes; elle pourrait bien l'être aussi par ses malheurs. Enfin c'est un fait inexpliquable au point de vue humain que ce spectacle d'une nation en grande majorité catholique asservie par une infime bande de cosmopolites, une poignée de juifs et de francs-maçons. (...) Sans doute la France comme peuple est restée chrétienne (...) mais la France officielle est un apostat. Et le sang des coupables retombe, c'est presque une loi de l'histoire, sur la tête des innocents qui sont les victimes ordinaires de l'expiation. ${ }^{10}$

Nous voyons que, dans l'esprit de Groulx, les dirigeants français anti-cléricaux ne représentent pas le peuple, qui les a pourtant élus; la seule explication possible de cette élection est donc un aveuglement suscité par Dieu pour punir le peuple qui a fait la Révolution contre son roi et contre l'Église. Nation ingrate, la France a perdu la protection de Dieu contre les menées diaboliques qui lui font choisir comme dirigeants des suppôts de Satan, dont les actes vont lui attirer le châtiment suprême, c'est-à-dire la mort, la disparition au sein d'un grand ensemble cosmopolite. La France, par son infidélité de 1789, s'est donc engagée dans un cycle infernal dont elle a peu de chances de s'échapper; fille aînée de l'Église, appelée au premier rang parmi les nations tant qu'elle restait fidèle au plan divin, elle risque comme l'ancien Israël de tomber d'autant plus bas qu'elle avait été élevée par le Créateur. Nous constatons donc que chez Groulx, les notions de patrie et de religion sont indissociables: les nations ont été créées par Dieu pour remplir une mission et elles sont condamnées à devenir des agrégats humains sans âme et sans avenir si elles s'en écartent; comme les individus, les peuples pécheurs sont voués à l'enfer représenté dans ce cas par un gouvernement de sans-patrie.

La France a forfait et s'est livrée aux «fils de Voltaire et de Julien l'Apostat ${ }^{11}$; ce fait est d'une exceptionnelle gravité puisque ce pays occupait le premier rang dans la hiérarchie des nations. Que va-t-il se passer? Nous trouvons la réponse dans un texte de 1898:

La mission de la France dans l'Europe n'est plus douteuse devant l'histoire. La France c'est l'Israël des temps nouveaux choisi par Dieu pour être le suprême boulevard de la foi du

10 Ibid., cahier V, $148 \mathrm{~ms}$.

11 Ibid., cahier V, $149 \mathrm{~ms}$. 
Christ venu, l'épée et le bouclier de la justice catholique. Et notre jeune pays, c'est un fils de la France. Le CanadaFrançais est donc héritier des privilèges de la mère-patrie. Dieu ne peut nous avoir déshérités d'un patrimoine qui nous revient légitimement: Dieu merci jamais rien de ce qui vit sous le ciel du Canada-Français n'a mérité que Dieu nous dépouille de nos droits privilégiés. Nous ne sommes point des félons. ${ }^{12}$

Contrairement à l'ancienne France qui a cessé d'être française en cessant d'être catholique, la Nouvelle-France est restée fidèle à la religion et à la vie rurale qui font la nationalité française authentique. Comme le disait Groulx dès 1896:

La Province de Québec au Canada, c'est la vieille Armorique, la terre bretonne de la France. (...) Nous sommes en Canada ce que les Bretons sont dans la France et c'est parmi nous que se conservent les saines traditions du passé et c'est à nous particulièrement que les ancêtres ont légué leur culte de la religion et de la patrie. ${ }^{13}$

C'est donc le Canada français, le Québec, qui hérite du droit d'aînesse de sả mère par sa fidélité aux traditions du passé; la nation québécoise est donc appelée à occuper le premier rang dans la hiérarchie des peuples.

La vision du monde de Groulx aboutit à une espérance messianique: le Québec sera dans le monde américain de demain ce que la France fut dans le monde européen et ce qu'Israël fut dans le monde antique. Dépassant les autres peuples par leur catholicisme, leur tradition française, leur vie rurale et leur jeunesse américaine, les Canadiens français sont le nouveau peuple élu porteur d'une mission civilisatrice en Amérique du Nord et par conséquent dans le monde entier. Le Québec est la nation parfaite et le chanoine Groulx se considérera et sera reçu comme le prophète de ce nouvel Israël, consacrant sa vie à la formation d'une élite qui actualisera les potentialités de la nation et qui la rendra capable d'assumer sa mission de régénération universelle.

Si le jeune séminariste a pu élaborer une telle vision du monde, c'est qu'elle correspondait à une expérience vécue: né à Vaudreuil dans un milieu catholique, francophone, rural et nord-américain, 
Groulx ne pouvait concevoir d'idéal ne réunissant pas ces quatre données pour lui indissociables. Né dans un milieu pré-industriel, donc peu enclin à s'ouvrir à l'autre sans le réduire à ses propres catégories, peu enclin à ne pas considérer ses valeurs comme la norme absolue, enfin peu enclin à ne pas se concevoir comme le centre du monde, Groulx ne pouvait faire graviter sa vision du monde qu'autour de son peuple, de son village, de sa personne même. En 1896, rêvant d'être oiseau pour fuir le collège où il étouffe, il écrit:

Si j'étais oiseau (...) j'irais voir les pays étrangers; j'allais même me poser sur la coupole de Saint Pierre à Rome. Et de temps à autre naturellement, je n'étais pas sans aller me reposer quelque peu sur mon petit clocher de Vaudreuil. ${ }^{14}$

Le raccourci est saisissant. D'un coup de plume, Groulx abolit toute la distance qui sépare Rome et son village natal; un peu plus et Vaudreuil deviendrait la nouvelle Rome, le centre du monde.

Avec une telle vision, comment Groulx allait-il réagir en arrivant dans la vraie Rome en 1906? Allait-il s'ouvrir à l'altérité, à la différence, ou au contraire allait-il ne rien voir qui ne fut déjà dans son imaginaire? C'est à cela qu'il faut tenter de répondre.

(Y.D.)

\section{1- Ses premiers contacts avec l'Europe (1906-1909)}

Cette Europe où il allait séjourner pendant près de trois ans (parti le 13 octobre $1906^{15}$, il est revenu au pays le 11 juillet 1909), Lionel Groulx, nous l'avons vu, la connaissait dès avant son départ. Nous pouvons d'ailleurs lire dans une lettre ${ }^{16}$ adressée à ses parents

14 Ibid., cahier I, $30 \mathrm{~ms}$.

15 Son départ pour Rome était prévu depuis longtemps. Nous pouvons lire dans une lettre que sa mère lui a adressée le 28 septembre 1902 (p. I ms.): «Je suis bien contente d'apprendre que tu sois bien traité (...) mais pour Rome, c'est un voyage trop long pour toi.» Ce n'est que le 11 janvier 1906 qu'il annonce officiellement à Émile Léger son départ pour Rome: «... Mgr me réservait pour étrennes mon «ticket of leave» pour Rome» (Lettre à Émile Léger, 11 janvier 1906, p. I ms.). Signalons finalement que l'organisation du voyage ne s'est pas faite sans quelques difficultés (comme le montrent les lettres à Émile Léger du 22 avril, 22 juin et 21 juillet 1906).

16 La correspondance de voyage de Lionel Groulx (15 octobre 1906-10 juillet 1909) que nous avons en main compte 50 correspondants dont deux anonymes et totalise 336 lettres et cartes postales. De ce nombre, 132 lettres furent envoyées par Groulx. Dans le choix de nos citations, nous nous sommes attachés aux dossiers qui nous semblaient complets: lettres à ses parents et lettres à Émile Léger. Notons que cette documentation se trouve à la Fondation Lionel-Groulx. 
en date du 24 octobre 1906: «Quand j'étudiais ces pays lointains dans ma géographie... $\rangle^{17}$

Non seulement jouissait-il d'une connaissance livresque approfondie de l'histoire et de la géographie de ces pays mais son opinion semblait déjà inébranlablement fixée ${ }^{18}$ sur les groupes humains et surtout sur les idées auxquelles il serait immanquablement confronté. Dans ce sens, et en faisant toutes les réserves qui s'imposent en pareil cas, nous pouvons affirmer que Lionel Groulx n'a jamais découvert l'Europe ailleurs que dans ses livres. En effet, la rhétorique de ses Notes et souvenirs de mon voyage en Europe ${ }^{19}$ nous invite à considérer ce premier voyage non pas, comme nous serions en droit de nous y attendre, comme un voyage de découvertes mais déjà comme un voyage de repérage de lieux et de mentalités avec lesquels il était familier.

Pour illustrer notre propos, nous examinerons deux textes de Lionel Groulx portant sur les catacombes que l'on retrouve dans ses Notes et souvenirs de mon voyage en Europe. Le premier de ces textes est daté du 18 novembre 1906 et se lit comme suit:

La vie catholique s'exhale encore des catacombes. Je ne les ai pas encore visitées. Mais je songe souvent à ces souterrains où vivaient les ancêtres du christianisme sous les pas des Empereurs tout-puissants, et des légions romaines qui ne doutaient plus que la conquête du monde pût leur être jamais ravie. Quelles leçons d'espérance à prendre en ces heures difficiles, dans la contemplation de ces vieux souvenirs, et quelle vitalité divine avait la foi qui, sortie de là, a pu faire les immenses choses qui ont été accomplies. ${ }^{20}$

17 Pages 1 et 2 de la lettre manuscrite. Dans une lettre à ses parents, en date du 19 octobre 1906 (il s'agit d'une lettre de 12 pages manuscrites, datée du 15 octobre, dans laquelle il relate au jour le jour ses impressions de la traversée), il écrit à la page 7 au sujet de l'île Florès de l'archipel des Açores: «Nous ne voyons rien bien-entendu (sic) puisque nous passons peut-être à cinq lieus (sic) du rivage. Seulement nous le savons par la géographie...». Précisons également qu'il indique fréquemment dans sa correspondance, les souvenirs de lecture que font renaître les lieux qu'il visite.

18 Lionel Groulx a par la suite réajusté son point de vue sur l'Europe, spécialement en ce qui concerne la France. Voir à ce sujet Mes Mémoires I: 373 et s. (deuxième voyage en Europe).

19 Ces notes sont consignées dans un cahier (81/4'” x 121/4"') dont seulement les 64 premières pages sont utilisées (une page est blanche). Il s'agit d'un journal de voyage dont le premier texte est daté du 4 novembre 1906 et le dernier du 25 novembre 1911 (les deux dernières pages sont occupées par des textes rédigés après son retour d'Europe). Ce journal inédit se trouve à la Fondation Lionel-Groulx.

20 Notes..., $11 \mathrm{~ms}$. 
Le deuxième texte fut écrit le 22 novembre 1906, le jour où il visita cet hypogée. Nous pouvons lire:

Et c'était là l'Église, l'Église de Jésus-Christ, c'étaient là les conquérant du monde; ceux qui venaient fermer le Colisée, ébranler le Palatin (...), rendre désert le Forum.

et plus loin:

...Quel acte de foi et quels élans d'espérance vous arrachent spontanément les effluves mystérieux qui sortent de ces murs sacro-saints, de ces dalles foulées par les pieds des apôtres et des martyrs! 21

Le parallélisme dans la structure des textes, les similitudes dans la rhétorique et dans la stylistique ainsi que le choix du lexique nous révèlent avec une étonnante netteté cette tendance volontaire à confirmer un jugement préalablement porté sur ce qu'il voyait. Les exemples du même type foisonnent autant dans la correspondance que dans les Notes et souvenirs de mon voyage en Furone. Il faut lire pour s'en convaincre davantage la description qu'il commet du monument de Garibaldi dans laquelle son inimitié contre le personnage motive sa critique sévère du travail sculptural:

Le monument a des proportions gigantesques, piédestal en granit, statue équestre en bronze. Le cheval ne parait (sic) bien qu'en face. Giuseppe Garibaldi a la mine pas du tout martiale d'un bon bourgeois qui ne sait pas même se tenir sur ses étriers. On lui fait regarder le Vatican d'un regard qui voudrait être foudroyant, et qui n'est que le froncement de sourcil d'un rentier qui voudrait jouer à l'homme de guerre. ${ }^{22}$

Puisque, pour cette période précise, il semble impossible de formuler l'épigénèse de l'idée que Groulx se faisait de l'Europe de laquelle il n'attendait rien qu'il ne connaissait déjà, on s'interroge sur les raisons qui ont motivé cet exil de trois ans, par moments fort pénible pour lui, comme en témoigne cette lettre du 23 décembre 1906 adressée à Émile Léger ${ }^{23}$ :

C'est une vie morne, lourde, qu'il fait bon de voir couler vite. Faites donc bonne provision d'endurance, de santé, de joies, de souvenirs du Canada avant de passer la mer. On ne saurait

21 Ibid., $13 \mathrm{~ms}$.

22 Ibid., $29 \mathrm{~ms}$

23 Disciple de Lionel Groulx à Valleyfield. Oncle du cardinal Paul-Émile Léger. Décédé prématurément le 22 juin 1908. 
trop se dire qu'on part pour ne pas revenir aux vacances prochaines. C'est incroyable comme on se sent loin, loin, loin. ${ }^{24}$

À la lumière des inédits de cette période, il semble que cette motivation s'articule autour de deux pôles: d'abord bien sûr, les études que Groulx entend compléter à Rome puisque, comme il le signale à Ėmile Léger dans sa lettre du 20 février 1908: «le temps vient où la soutane du prêtre toute seule ne vaudra plus un certificat de compétence ès éducation ${ }^{25}$; puis, comme nous l'avons entrevu précédemment, la confirmation du bien-fondé d'un système déjà en place avant son séjour. À ce sujet et plusieurs années plus tard, le Chanoine nous livre dans ses Mémoires avec un naturel intact cet état d'étanchéité de sa pensée à son arrivée en Europe en 1906: «... à mon départ pour l'Europe en 1906, mon système d'idées, si jamais je me suis fabriqué quelque chose de cette sorte, était passablement arrêté. ${ }^{26} \mathrm{Il}$ va sans dire que, dans cette optique, ce n'est pas la réalité qui produit la théorie mais la théorie (son système) qui interprète une certaine réalité.

Ce système qu'il a par la suite affiné pendant 61 ans était alors soutenu par quatre axes principaux: le catholicisme, la francophonie, la vie rurale et l'Amérique. La confirmation d'un catholicisme inébranlable, Groulx l'a évidemment trouvée à Rome où il renifle allègrement, suivant ses mots, «le parfum religieux (...) dans la ville du Pape» ${ }^{27}$. Dans ses Notes et souvenirs de mon voyage en Euro$p e^{28}$, il distingue trois sources d'exhalation de la vie catholique à Rome: d'abord les basiliques et les églises où reposent saints et martyrs et dont l'architecture symbolise à ses yeux la pérennité de la foi chrétienne; puis les catacombes qui représentent l'espérance dans la foi pour la conquête du monde; finalement les monuments de la Rome païenne, ces «débris antiques» ${ }^{29}$ comme il les désigne, dont le lamentable état des structures symbolise la défaite du monde païen aux mains du monde chrétien. Mais par-dessus tout, Rome est la cité papale. Son audience avec Pie X demeure «le plus grand souvenir de ma vie», avouera-t-il à Émile Léger dans sa lettre du 29 jan-

24 Page $2 \mathrm{~ms}$.

25 Pages 3 et 4 ms.

26 Lionel Groulx. Mes Mémoires (Fides, 1970), I: 154.

27 Lettre à Emile Léger, 14 novembre 1906, $4 \mathrm{~ms}$.

28 Notes..., 10-11 ms.

29 Ibid., $6 \mathrm{~ms}$. 
vier $1907^{30}$. Groulx n'a par la suite jamais caché son admiration pour le pontificat de Pie $\mathrm{X}^{31}$. Dans la lettre précitée, il déclare avec toute l'exaltation qu'on lui connaît: «Quel Pape que Pie X. Sa dernière encyclique aux Français $m$ 'a remué si fortement que je vénère en lui l'un des plus grands papes de l'Église. $)^{32}$ De Rome, il ne retiendra guère autre chose que ce sentiment profond d'exaltation du catholicisme. C'est ainsi que nous pouvons lire dans cette lettre du 29 janvier à Émile Léger:

...arrangez-vous pour venir à Rome, la ville où, à défaut de quelque chose de mieux, on prend le sens catholique, et un amour profond et suprême de l'Église. Voilà ce que j'aurai rapporté de meilleur de mon séjour dans la cité du Pape. ${ }^{33}$

Quant à la langue française, il la retrouve en France, utilisée pour la propagation de l'anticléricalisme ${ }^{34}$ qui triomphe avec Combes et Clémenceau. Paris le déçoit donc amèrement. La Bretagne, par contre, le réconcilie avec la mère patrie. Il y retrouve dans un décor chlampêtre ́́ce qui ne manquait pas de le àisposer fávorabobement) une vie catholique centrée sur une autre personnalité, celle de l'Amiral de Cuverville ${ }^{35}$, noble légitimiste auprès duquel il remplit les fonctions d'aumônier.

Mais Groulx se lasse de cette Europe tiraillée où il résiste à toute forme d'acculturation et c'est à l'image de son Canada français qu'il songe sans arrêt. Il confiera à Émile Léger le 23 décembre 1906: «Le voyage en Europe est beau envisagé dans ses suites, et raisonné au point de vue de l'avenir. ${ }^{36} \mathrm{C}^{3}$ 'est ainsi qu'à mesure que le temps progresse, il écrit de moins en moins sur l'Europe dans sa correspondance pour finalement n'en parler à peu près plus. Autre indice, son journal de voyage ne compte que 63 pages manuscrites et couvre une période de trois ans. Pour une période équivalente, son journal intime, rédigé au Québec, peut s'évaluer à 220 pages.

30 Page $4 \mathrm{~ms}$. Voir aussi: Lettre à ses parents, 25 janvier 1907, 1 ms. et les Notes..., 25 et s. ms.

31 Lionel Groulx. Mes Mémoires, op. cit., 117-119.

32 Page $4 \mathrm{~ms}$.

33 Pages 3 et 4 ms.

34 Dans la correspondance de voyage de Groulx, la France est le plus souvent jugée en regard de son anticléricalisme. Nous donnerons en exemple cette affirmation tirée d'une lettre adressée à ses parents le 20 août 1907 (p. 3 ms.): «Ä vouloir se passer de Dieu et de l'Église, les Français gagnent, ce qui arrive toujours, de devenir un peuple de corrompus"».

35 Nous pouvons retrouver 7 lettres de l'Amiral de Cuverville à Lionel Groulx (entre le 11 juillet 1906 et le 11 mai 1909) à la Fondation Lionel-Groulx.

36 Page $2 \mathrm{~ms}$. 
Cette volonté de mieux comprendre son Canada français par le biais de l'Europe, de le retrouver par le détour de cette sorte de palimpseste idéologique, déborde en dernière analyse la stricte préoccupation des questions internationales. Nous pouvons observer ce revirement dans la description qu'il donne d'une statue de Jeanne d'Arc:

La statue de l'Hôtel de Ville représente Jeanne comme dans l'attitude de la prière les mains jointes sur la garde de son épée qu'elle presse sur sa poitrine. Une leçon à tirer: jeunes gens qui priez pour votre pays, priez vous aussi en pressant une arme, votre plume, l'épée des paladins d'aujourd'hui, pour que vous méritiez d'être choisis par Dieu parmi ceux qui bouteront l'ennemi hors de la Nouvelle-France. ${ }^{37}$

À l'instar du Journal intime, les écrits de voyage de Groulx inscrivent sa vision du monde dans cette espérance messianique où le Canada français prendrait la relève d'une France déchue, décatie, et serait investi du rôle de civilisateur chrétien non seulement en Amérique mais dans le monde entier. La nation canadiennefrançaise semble la seule à ses yeux qui puisse répondre aux quatre exigences du système, en sa qualité de nation catholique, française, à vocation rurale et située dans un monde neuf, l'Amérique. C'est ainsi qu'il dit à ses parents dans une lettre du 25 janvier 1907: «...l'on est encore mieux à cultiver la terre dans la petite province de Québec qu'à être roi en Europe.»» ${ }^{38}$

Voilà rapidement présentées, quelques constantes de cette pensée à caractère allotropique que nous profilent les inédits de Groulx pendant son premier voyage en Europe.

(R.B.)

$37 \quad$ Notes..., $59 \mathrm{~ms}$.

38 Page $4 \mathrm{~ms}$. Nous pouvons encore lire dans une lettre à ses parents, datée du 15 juillet 1907 (p. 2 ms.): «Les souvenirs du pays nous suivent et me suivent pour ma part, partout où je vais. Je vous estime bien heureux d'y vivre. Et cette pensée doit vous consoler de ne pas voir les grandes choses que je vois, mais qui ont le grand tort de se faire voir en pays étrangers.» Dans une autre lettre envoyée à ses parents le 6 juillet 1907 (p. I ms.), il propose ce curieux aménagement de la géographie: «Le pays est ici magnifique (il est à Assise), et le serait à ravir si l'on pouvait transporter au Canada, ces montagnes et toutes ces plaines de l'Ombrie baignées de douceur et de lumière bleue.» 